\title{
Microcystins do not provide anti-herbivore defence against mixotrophic flagellates
}

\author{
Susanne Wilken ${ }^{1,2, *}$, Suzanne Wiezer ${ }^{1}$, Jef Huisman ${ }^{2}$, Ellen Van Donk ${ }^{1,3}$ \\ ${ }^{1}$ Department of Aquatic Ecology, Netherlands Institute of Ecology, Rijksstraatweg 6, 3631 AC Nieuwersluis, The Netherlands \\ ${ }^{2}$ Aquatic Microbiology, Institute for Biodiversity and Ecosystem Dynamics, University of Amsterdam, Nieuwe Achtergracht 127, \\ 1018 WS Amsterdam, The Netherlands \\ ${ }^{3}$ Palaeoecology, Institute of Environmental Biology, University of Utrecht, Padualaan 8, 3584 CH Utrecht, The Netherlands
}

\begin{abstract}
While most experiments investigating zooplankton grazing on harmful cyanobacteria have been carried out with metazoan plankton, several protozoa can also feed efficiently on cyanobacteria. We investigated grazing by the mixotrophic flagellate Ochromonas sp. on the toxic cyanobacterium Microcystis aeruginosa. Ochromonas sp. grew rapidly on $M$. aeruginosa and had a strong impact on the population density of its prey. However, specific growth rates of Ochromonas sp. decreased over time, possibly indicating a negative impact on Ochromonas sp. mediated by $M$. aeruginosa. Grazing did not have any effect on the intracellular microcystin content of $M$. aeruginosa, and the ingested microcystins did not accumulate within Ochromonas sp. We studied the functional and numerical response of Ochromonas sp. grazing on the microcystin-producing strain $M$. aeruginosa PCC 7806 and its microcystin-deficient mutant. Ochromonas sp. showed a Type 3 functional response of very similar shape on both the toxic and non-toxic $M$. aeruginosa strain. Ingestion rates of Ochromonas sp. were even slightly higher on the toxic $M$. aeruginosa strain. We therefore found no indication of microcystins acting as a defence against mixotrophic flagellates.
\end{abstract}

KEY WORDS: Microcystis · Microcystins · Mixotrophic flagellates · Ochromonas sp. · Grazing Resale or republication not permitted without written consent of the publisher

\section{INTRODUCTION}

Cyanobacterial blooms have increasingly become a nuisance in many freshwater and brackish ecosystems (Chorus \& Bartram 1999, Carmichael 2001, Huisman et al. 2005). They are facilitated by high water temperatures and nutrient load and therefore benefit from anthropogenic influences like eutrophication and climate warming (Codd 2000, Jöhnk et al. 2008, Paerl \& Huisman 2008). Microcystin-producing cyanobacteria of the Microcystis genus are widely distributed bloomforming species. The hepatotoxic microcystins inhibit protein phosphatases in eukaryotes (MacKintosh et al. 1990) and are therefore toxic to many potential grazers of Microcystis (Fulton \& Paerl 1987b, DeMott et al. 1991). Other factors contributing to the low edibility of Microcystis are the formation of colonies leading to interference with the feeding behaviour of many zooplankton species (Fulton \& Paerl 1987a, Yang et al.
2006) and the low content of long-chain polyunsaturated fatty acids and sterols, which are essential for many herbivores (Wacker \& von Elert 2001, von Elert et al. 2003).

While most experiments investigating effects of toxic cyanobacteria on pelagic grazers have been done with metazoans, several protozoa are able to maintain high growth rates when feeding on cyanobacteria including Microcystis (Cole \& Wynne 1974, Nishibe et al. 2002, Kim et al. 2006). Especially mixotrophic chrysophytes, such as Ochromonas spp. and Poterioochromonas spp., can graze efficiently on Microcystis and even degrade the cyanotoxins (Ou et al. 2005, Zhang et al. 2008, Van Donk et al. 2009). The genus Ochromonas is abundant in freshwaters and has been reported to frequently cooccur with Microcystis (Van Donk et al. 2009). Ochromonas has therefore been proposed as a potential biological control agent against Microcystis blooms (Cole \& Wynne 1974, Zhang et al. 2008), although little is 
thus far known about its interaction with toxic cyanobacteria. As mixotrophs, these organisms differ considerably from purely heterotrophic grazers by their photosynthetic machinery and the presence of several biochemical pathways typical of autotrophic organisms. For instance, in contrast to many heterotrophs, Ochromonas is able to synthesize long-chain polyunsaturated fatty acids (Boëchat et al. 2007) and thus does not depend on the presence of these substances in its prey. However, Ochromonas might be susceptible to the other 2 mechanisms of grazer deterrence in Microcystis, e.g. colony formation and microcystin production. Colony formation in Microcystis can be induced by the presence of Ochromonas sp. (Burkert et al. 2001, Yang et al. 2006). Even though Ochromonas is able to ingest surprisingly large prey items, there is certainly an upper limit of suitable prey size, and inducible colony formation is likely to be an efficient way of defence against grazing by flagellates.

In contrast to colony formation, the role of microcystin production as a defence against flagellate grazers is less clear. The original function of microcystins is still under debate, and its role as a defence against grazers has been questioned, because the genes for microcystin biosynthesis seem to be older than metazoan grazers such as copepods and cladocerans (Rantala et al. 2004). However, since the earliest protozoa probably already preyed upon cyanobacteria long before the metazoans evolved, the early evolution of microcystins does not necessarily exclude its original role as a defence against protozoa. Furthermore, the function of these secondary metabolites might have changed during their evolution, and regardless of the original function of microcystins they are currently toxic for many species. Ochromonas could pay a metabolic cost for its ability to grow on toxic cyanobacteria and to degrade the toxins, which might be reflected in lower growth rates compared to growth on non-toxic food. Ou et al. (2005) described an initially stimulating effect of dissolved microcystins on the growth of Poterioochromonas sp., but decreased growth rates after prolonged exposure to the toxins. This suggests that microcystins might indeed act as a defence against grazing by protozoan flagellates. Grazer-induced defences are well known for several phytoplankton taxa, such as the induction of colony formation in the green alga Scenedesmus (Lürling \& Van Donk 1997). Jang et al. (2003) reported an increased microcystin content in Microcystis as a response to Daphnia sp. grazing, suggesting the microcystin production of cyanobacteria to be induced by info-chemicals released by the grazer. Whether the microcystin production of Microcystis responds to flagellate grazing is not known yet, however.

Here, we investigate the functional and numerical response of the mixotrophic flagellate Ochromonas sp. grazing on the microcystin-producing cyanobacterium Microcystis aeruginosa PCC 7806 and its microcystindeficient mutant. Conversely, we also investigate whether flagellate grazing induces an enhanced microcystin production by $M$. aeruginosa.

\section{MATERIALS AND METHODS}

Phytoplankton strains. We investigated the microcystin-producing cyanobacterium Microcystis aeruginosa PCC 7806, its microcystin-deficient mutant (Dittmann et al. 1997) and the microcystin-producing strain $M$. aeruginosa HUB 524. All 3 M. aeruginosa strains were single celled and did not show any colony formation in the stock cultures. Our Ochromonas sp. strain was detected as contamination in large-scale mesocosm experiments with $M$. aeruginosa. It was isolated by micro-needle techniques. Stock cultures of all strains were maintained on COMBO-medium (Kilham et al. 1998). For Ochromonas sp., ammonium was used as the nitrogen source in the stock cultures, because the strain grew rather poorly on nitrate. All cultures were unialgal, but not axenic. Abundances of heterotrophic bacteria were low, however, and never exceeded $1 \%$ of the total biovolume. Cellular microcystin contents and cell volumes of the strains are summarized in Table 1.

Effect of grazing on toxin production. We investigated effects of grazing on the microcystin production of Microcystis aeruginosa PCC 7806 and M. aeruginosa HUB 524. The experiments were done in batch cultures using $500 \mathrm{ml}$ Erlenmeyer flasks filled with $300 \mathrm{ml}$ COMBO-medium (Kilham et al. 1998). We applied 4 experimental treatments to each $M$. aeruginosa strain: (1) monocultures of $M$. aeruginosa that served as controls, (2) monocultures of $M$. aeruginosa

Table 1. Microcystis aeruginosa and Ochromonas sp. Microcystin content ( $\mathrm{n}=$ 16) and biovolume $(\mathrm{n}=3)$ of the $M$. aeruginosa strains and Ochromonas sp. used in the experiments. Standard deviations are given in parentheses. Measurements reported in this table were made on Day 0 of the grazing experiment

\begin{tabular}{|lcc|}
\hline Strain & $\begin{array}{c}\text { Microcystin content } \\
\left(\mathrm{fg} \mathrm{cell}^{-1}\right)\end{array}$ & $\begin{array}{c}\text { Biovolume } \\
\left(\mu \mathrm{m}^{3} \mathrm{cell}^{-1}\right)\end{array}$ \\
\hline M. aeruginosa PCC 7806 wildtype & $53.32( \pm 6.66)$ & $25.02( \pm 1.64)$ \\
M. aeruginosa PCC 7806 mutant & - & $28.10( \pm 3.07)$ \\
M. aeruginosa HUB 524 & $27.16( \pm 3.00)$ & $32.08( \pm 0.14)$ \\
Ochromonas sp. & - & $146.57( \pm 30.91)$ \\
\hline
\end{tabular}


to which we added the filtrate of an Ochromonas sp. culture pre-fed with the same $M$. aeruginosa strain, (3) monocultures of $M$. aeruginosa to which we added the filtrate of an Ochromonas sp. culture pre-fed with a different prey species, the Eustigmatophyte Nannochloropsis limnetica, and (4) mixed cultures of $M$. aeruginosa and Ochromonas sp. that served as grazing treatment. All 4 treatments were performed in 4 replicates. The experiments were incubated at $23^{\circ} \mathrm{C}$ with a low shaking frequency of $20 \mathrm{rpm}$ and an incident light intensity of $90 \mu \mathrm{mol}$ photons $\mathrm{m}^{-2} \mathrm{~s}^{-1}$ under a $14 \mathrm{~h}$ light:10 h dark photocycle.

In all treatments, we inoculated Microcystis aeruginosa to a final population density of $3 \times 10^{5} \mathrm{cells} \mathrm{m}^{-1}$. In the species mixture of the fourth treatment, Ochromonas sp. was added to a final population density of 500 cells $\mathrm{ml}^{-1}$. These rather low population densities were chosen to prevent $M$. aeruginosa from reaching the stationary phase in the control while it was still growing exponentially in the grazing treatment. Such a situation might lead to differences in microcystin concentration caused by differences in growth rates, nutrient status (Long et al. 2001), or light intensity (Wiedner et al. 2003) between the grazing treatment and the control that would not be directly related to the grazing itself. Our inoculation densities were lower than $M$. aeruginosa abundances in dense surface blooms, but comparable to the abundances of both species in natural waters during non-bloom conditions (Van Donk et al. 2009). The filtrates originated from rather dense cultures of Ochromonas sp. pre-fed with Nannochloropsis limnetica and $M$. aeruginosa. The filtrates were added daily in volumes corresponding to the density of Ochromonas sp. in the grazing treatment, adding up to $25 \%$ of the culture volume over the course of the experiment. Equal volumes of fresh medium were added to the control and grazing treatments each time. Based on preliminary experiments, samples for population densities and microcystin analysis were taken every 2 to $3 \mathrm{~d}$ over a period of $9 \mathrm{~d}$.

In the first set of experiments, with Microcystis aeruginosa PCC 7806, population densities were measured directly after sampling using a CytoSense flow cytometer (CytoBuoy b.v.). In the second set of experiments, with $M$. aeruginosa HUB 524, samples were fixed with a mixture of glutaraldehyde and formaldehyde (final concentrations of 0.025 and 0.0037 percent by mass, respectively) and measured at a flow cytometer (MoFlo XDP Cell Sorter, Beckman Coulter) after a few days of storage at $4^{\circ} \mathrm{C}$. Fresh samples were regularly checked for colony formation by light microscopy, but $M$. aeruginosa remained single celled in both experiments.

Microcystin concentrations were measured using an enzyme-linked immunosorbent assay (ELISA) (QuantiPlate Kit for Microcystins; EnviroLogix). For measure- ment of particulate microcystins, the samples were centrifuged and the pellet was resuspended in MilliQ water. Samples were diluted to a final cell density of about $5 \times 10^{4}$ cells $\mathrm{ml}^{-1}$ to ensure that the microcystin concentration fell within the measurable range of the ELISA. Microcystins were extracted from the cells by 3 freeze and thaw cycles, each of which was followed by sonification for 15 min using a sonic bath (Gustafsson et al. 2005). For the measurement of dissolved microcystins, samples were filtered through glass fibre filters (Whatman GF/C), and the filtrate was analysed for microcystins. To distinguish between microcystins in Microcystis aeruginosa and Ochromonas sp. cells, we sorted $2.5 \times 10^{5} \mathrm{M}$. aeruginosa cells from the mixed cultures using a flow cytometer (MoFlo XDP Cell Sorter, Beckman Coulter). The sorted fraction containing only $M$. aeruginosa cells was diluted, and population density and microcystin content were measured using the same methods as for the other samples (CytoSense for the first experiment and MoFlo for the second experiment). Because population densities of Ochromonas sp. were too low for flow cytometer sorting throughout most of the experiment, it could only be sorted at the last day.

When variances were homogenous, the data were tested for a treatment effect by repeated-measures ANOVA, followed by a Tukey test as a post hoc comparison. When variances remained heterogeneous even after logarithmic transformation (microcystin data of PCC 7806), the non-parametric Kruskal-Wallis ANOVA was used instead. All statistical analyses were done using STATISTICA 8 software.

Functional and numerical response experiment. To investigate effects of microcystins on Ochromonas sp., the functional and numerical response of Ochromonas sp. grazing on the toxic strain Microcystis aeruginosa PCC 7806 and its microcystin-deficient mutant were compared. Ochromonas sp. was pre-cultured for $1 \mathrm{wk}$ on the 2 strains at prey concentrations used in the experiment. During pre-culturing, prey concentrations were measured daily using a CytoSense flow cytometer and readjusted to the desired concentrations by either dilution or addition of more prey. Stock cultures were harvested by centrifugation and resuspended in fresh COMBO-medium to prevent any potential effect of 'spent' medium. To assess the functional and numerical response of Ochromonas sp., we used 8 different concentrations of $M$. aeruginosa (Table 2). This included a treatment without any $M$. aeruginosa as food, to estimate the specific growth rate of Ochromonas sp. when grown photo-autotrophically. At each $M$. aeruginosa concentration, we had a treatment with Ochromonas sp. grazing and a control for $M$. aeruginosa growth without Ochromonas sp. All treatments were run in triplicate. The experiment was performed 
Table 2. Microcystis aeruginosa and Ochromonas sp. Initial population densities used in the functional and numerical response experiment

\begin{tabular}{|c|c|c|}
\hline $\begin{array}{l}\text { Treatment } \\
\text { no. }\end{array}$ & $\begin{array}{l}\text { M. aeruginosa } \\
\left(\text { cells } \mathrm{ml}^{-1}\right)\end{array}$ & $\begin{array}{l}\text { Ochromonas sp. } \\
\quad\left(\text { cells ml }{ }^{-1}\right)\end{array}$ \\
\hline 1 & 0 & $3.9 \times 10^{4}$ \\
\hline 2 & $2.6 \times 10^{3}$ & $5.0 \times 10^{2}$ \\
\hline 3 & $8.0 \times 10^{3}$ & $8.4 \times 10^{2}$ \\
\hline 4 & $2.4 \times 10^{4}$ & $2.9 \times 10^{3}$ \\
\hline 5 & $8.0 \times 10^{4}$ & $7.3 \times 10^{3}$ \\
\hline 6 & $2.5 \times 10^{5}$ & $2.5 \times 10^{4}$ \\
\hline 7 & $8.2 \times 10^{5}$ & $7.3 \times 10^{4}$ \\
\hline 8 & $2.7 \times 10^{6}$ & $1.2 \times 10^{5}$ \\
\hline
\end{tabular}

in $100 \mathrm{ml}$ Erlenmeyer flasks under continuous illumination of $90 \mu \mathrm{mol}$ photons $\mathrm{m}^{-2} \mathrm{~s}^{-1}$. Cultures were incubated at $23^{\circ} \mathrm{C}$ for $3 \mathrm{~d}$, and samples were taken at 0 , $3,6,12,24,48$ and $72 \mathrm{~h}$ after inoculation, fixed with a mixture of glutaraldehyde and formaldehyde as described in 'Effect of grazing on toxin production' and stored at $4^{\circ} \mathrm{C}$ until measurement with a flow cytometer (MoFlo XDP Cell Sorter, Beckman Coulter).

Because Ochromonas sp. might ingest heterotrophic bacteria as well, and our cultures were not axenic, we performed bacterial counts on several samples. We picked samples from Treatments 3,5 and 7 (Table 2) taken at 0 and $12 \mathrm{~h}$ after inoculation, for both the wildtype and the mutant of Microcystis aeruginosa PCC 7806. Bacteria were stained with SYBR Green (Invitrogen) prior to counting with the MoFlo flow cytometer.

Population densities were plotted against time on a semi-log scale. During periods in which a linear relation was observed, the specific growth rate, $\mu$, was calculated as:

$$
\mu=\frac{\ln \left(N_{2}\right)-\ln \left(N_{1}\right)}{t_{2}-t_{1}}
$$

where $N_{1}$ and $N_{2}$ denote population densities at time $t_{1}$ and $t_{2}$, respectively. Clearance rates were calculated over the first $3 \mathrm{~h}$ of incubation, except for the lowest 2 prey concentrations for which changes in concentrations were low enough to use a longer time span. The following formula was used (Heinbokel 1978):

$$
C l=\frac{\mu_{\mathrm{Mc}}-\mu_{\mathrm{Mt}}}{N_{\mathrm{O}}}
$$

where $\mathrm{Cl}$ is the clearance rate, $\mu_{\mathrm{Mc}}$ and $\mu_{\mathrm{Mt}}$ are the specific growth rates of Microcystis aeruginosa in the control and the grazing treatments, respectively, and $N_{\mathrm{O}}$ is the mean Ochromonas sp. abundance over the period used for calculation. Ingestion rates are then given by:

$$
I=N_{\mathrm{M}} C l
$$

where $I$ denotes the ingestion rate and $N_{\mathrm{M}}$ denotes the mean $M$. aeruginosa abundance.
Ochromonas sp. showed a slightly sigmoid increase of the ingestion rate and an initial increase of the clearance rate with increasing Microcystis aeruginosa abundance. This is indicative of a Type 3 functional response, where Ochromonas sp. increases its feeding rate with increasing prey density (Holling 1959, Kiørboe 2008). Therefore, we fitted a Type 3 functional response model to the ingestion rate data (Real 1977):

$$
I=\frac{I_{\max } N_{M}^{x}}{K^{x}+N_{M}^{x}}
$$

where $I_{\max }$ is the maximum ingestion rate reached at saturating prey concentrations, the power $x$ describes the curvature of the sigmoid functional response (we note that, if $x=1$, the model simplifies to a Type 2 functional response) and $K$ is the half-saturation constant, representing the prey density at which half the maximum ingestion rate is reached. The model parameters were estimated by non-linear regression, using the iterative least-squares method of STATISTICA 8. The ingestion-rate data were log-transformed prior to the model fit to improve the homogeneity of variance.

\section{RESULTS}

\section{Effect of grazing on toxin production}

Both Microcystis aeruginosa strains showed positive growth rates in all treatments, reaching population densities of 4 to $10 \times 10^{6}$ cells $\mathrm{ml}^{-1}$ at the end of the experiments (Fig. 1). Nevertheless, the presence of Ochromonas sp. had a strong effect on $M$. aeruginosa densities, reducing $M$. aeruginosa PCC 7806 by $44 \%$ and $M$. aeruginosa HUB 524 by $39 \%$ relative to the controls (repeated-measures ANOVA, for PCC 7806: $F_{3,12}=$ 36.37, $\mathrm{p}<0.001$; for HUB 524: $F_{3,12}=48.08$, $\mathrm{p}<0.001$ ). The addition of Ochromonas sp. filtrates did not show any effect on $M$. aeruginosa abundances. Ochromonas sp. grew rapidly on both strains, increasing its population density by almost 1000-fold over the course of the experiments (Fig. 1C,D). It reached its highest specific growth rates of $2.1 \mathrm{~d}^{-1}$ (on PCC 7806) and $1.5 \mathrm{~d}^{-1}$ (on HUB 524) at the beginning of the experiment, followed by a decrease of its specific growth rates to $0.21 \mathrm{~d}^{-1}$ (on PCC 7806) and $0.36 \mathrm{~d}^{-1}$ (on HUB 524) by the end, even though prey densities increased (Fig. 2).

The total particulate microcystin concentrations showed a similar increase as the population densities of Microcystis aeruginosa, reaching concentrations of

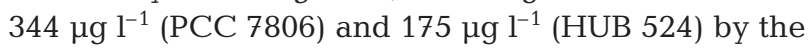
last day in the controls, and about $32 \%$ (PCC 7806) and $18 \%$ (HUB 524) lower values in the grazing treatment with Ochromonas sp. (Fig. 3A,B). The treatment effect was significant for $M$. aeruginosa HUB 524 (repeated- 

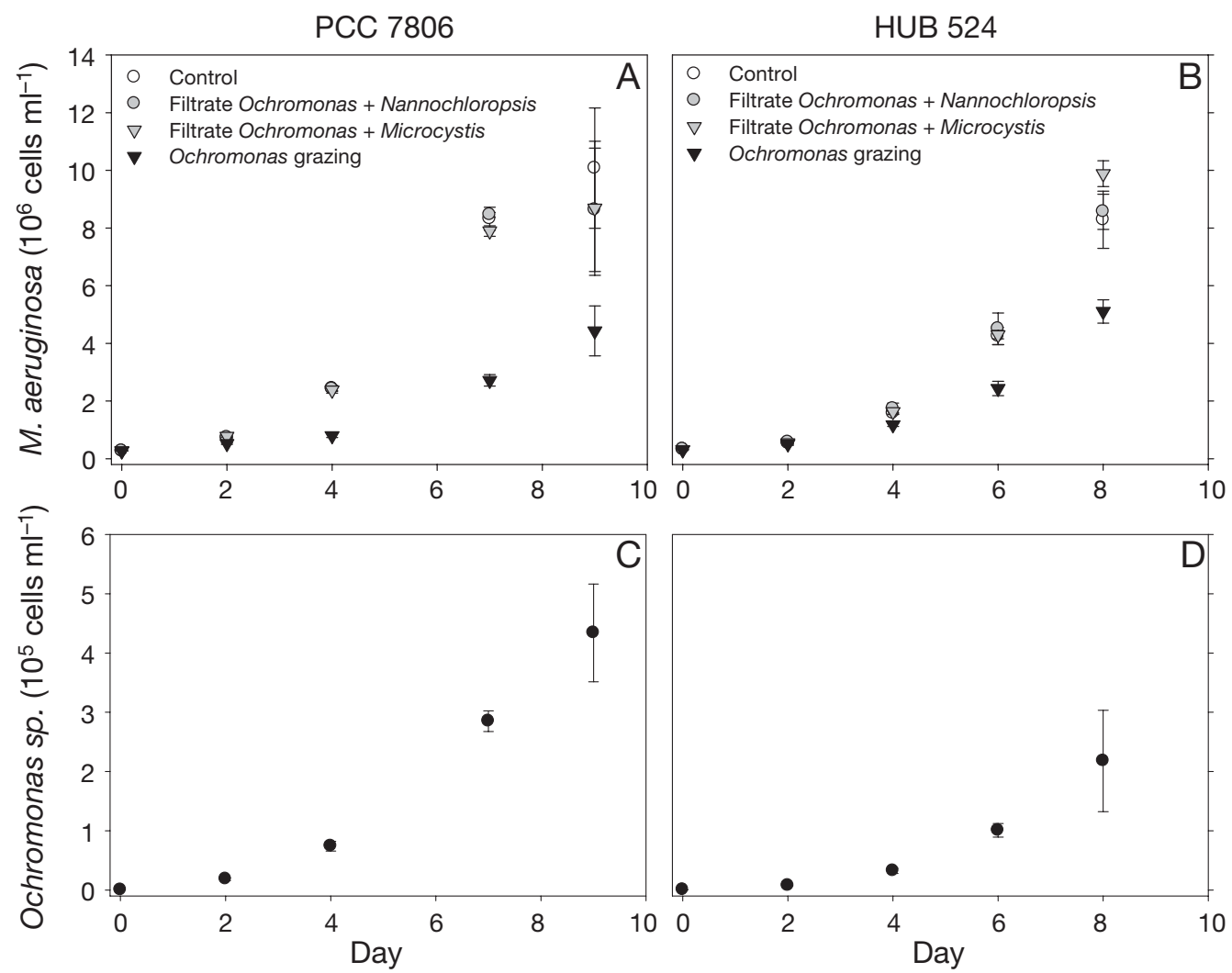

Fig. 1. (A,B) Microcystis aeruginosa and (C,D) Ochromonas sp. Population densities over the course of the grazing experiments with Ochromonas sp. as predator and (A,C) M. aeruginosa PCC 7806 and (B,D) M. aeruginosa HUB 524 as prey. Error bars indicate standard deviation

measures ANOVA, $F_{3,12}=8.03, \mathrm{p}<0.01$ ) and for $M$. aeruginosa PCC 7806 on Days 4 and 7 (Kruskal-Wallis ANOVA, $H_{3}=9.53, \mathrm{p}<0.01$ and $H_{3}=8.49, \mathrm{p}<0.05$, respectively). The intracellular microcystin content of M. aeruginosa PCC 7806 was about twice as high as

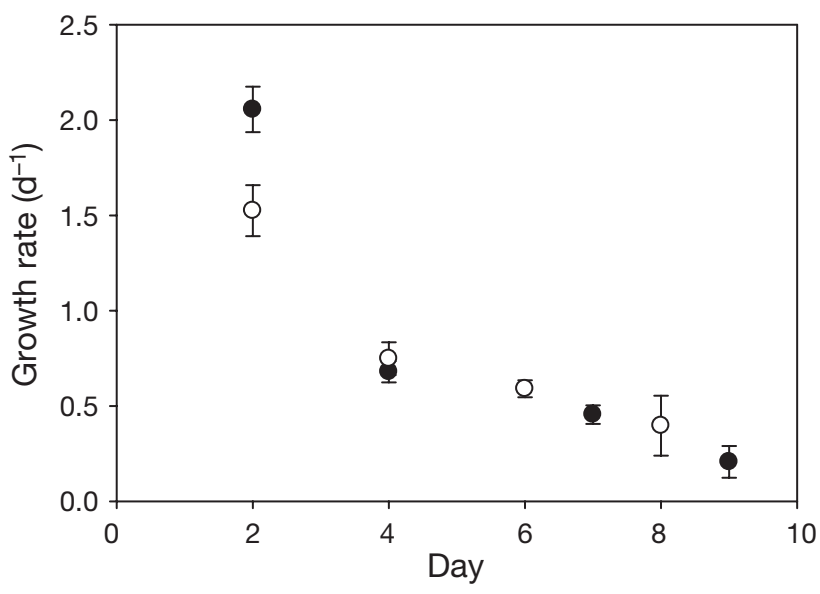

Fig. 2. Ochromonas sp. Specific growth rates of Ochromonas sp. on Microcystis aeruginosa PCC 7806 (filled circles) and M. aeruginosa HUB 524 (open circles) over the course of the grazing experiment. Error bars indicate standard deviation that of $M$. aeruginosa HUB 524 (Fig. 3C,D). The intracellular microcystin contents of both strains remained constant across all treatments (Fig. 3C), except at the end of the experiment with $M$. aeruginosa PCC 7806, where it fell to significantly lower values in the grazing treatment by Day 9 (Kruskal-Wallis ANOVA, $H_{3}=8.74$, $\mathrm{p}<0.05)$. Dissolved microcystin concentrations were low compared to the particulate concentrations, exceeding $5 \%$ of the total microcystins only for $M$. aeruginosa PCC 7806 on Day 9. Dissolved microcystin concentrations increased with increasing population densities of $M$. aeruginosa, and reached considerably higher concentrations for $M$. aeruginosa PCC 7806 than for $M$. aeruginosa HUB 524 (Fig. 3E,F). From Day 4 onwards, the dissolved microcystin concentrations were significantly higher in the presence than in the absence of Ochromonas sp. (repeated-measures ANOVA, for PCC 7806: $F_{3,12}=16.90, \mathrm{p}<0.001$; for HUB 524: $\left.F_{3,12}=61.78, \mathrm{p}<0.001\right)$. The intracellular microcystin content of Ochromonas sp. could be measured only at the end of the experiments, when population densities of Ochromonas sp. were high enough to be sorted by the flow cytometer. Ochromonas sp. contained $3.91( \pm 3.19 \mathrm{SD}) \mathrm{fg}$ microcystin cell ${ }^{-1}$ after $9 \mathrm{~d}$ 

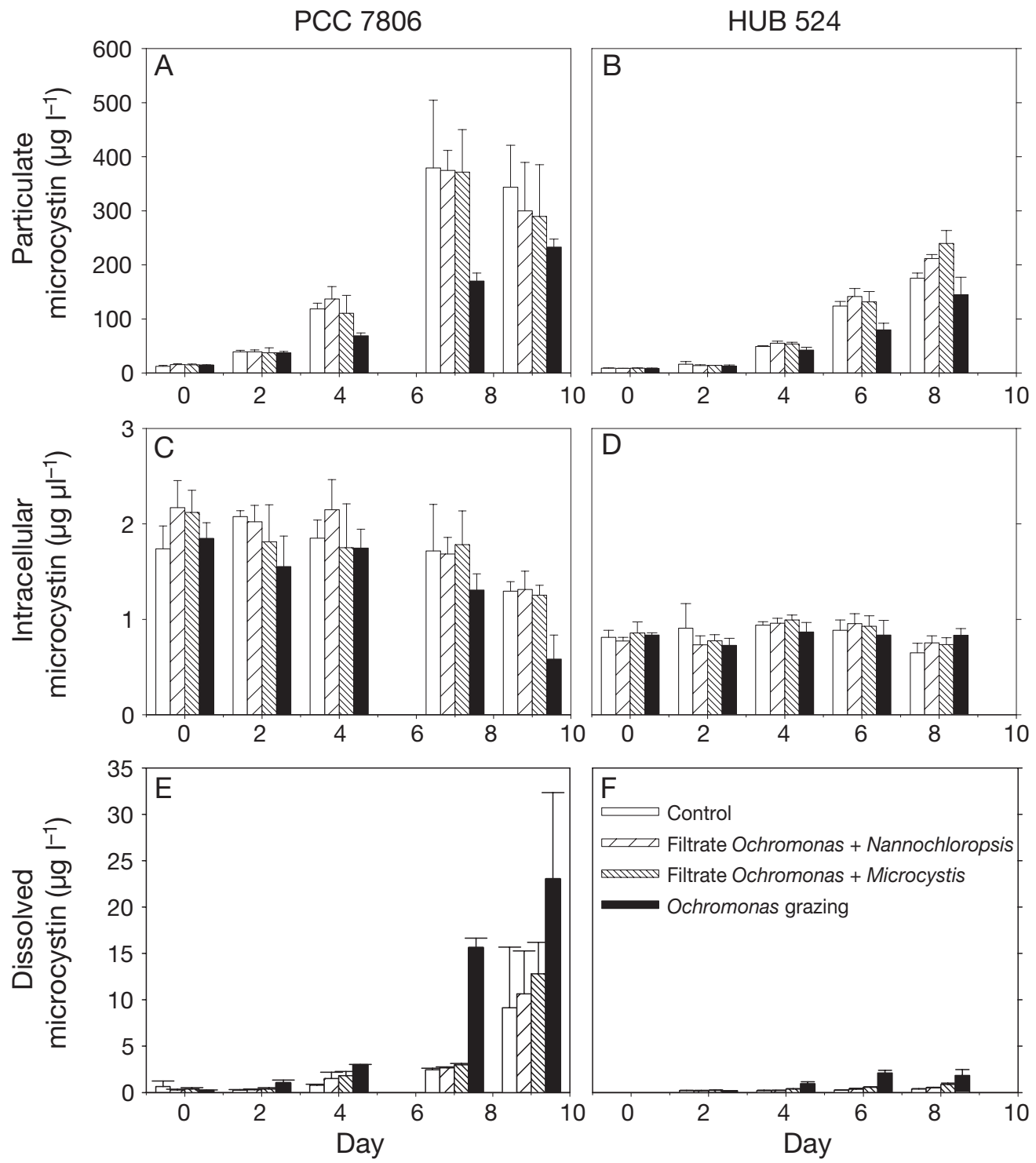

Fig. 3. Microcystis aeruginosa. Changes in $(\mathrm{A}, \mathrm{B})$ particulate microcystin concentration, $(\mathrm{C}, \mathrm{D})$ intracellular microcystin content and $(\mathrm{E}, \mathrm{F})$ dissolved microcystin concentration over the course of the grazing experiments with Ochromonas sp. as predator and M. aeruginosa PCC 7806 (left panels) and M. aeruginosa HUB 524 (right panels) as prey. Error bars indicate standard deviation

of grazing on $M$. aeruginosa PCC 7806 and 5.23 $( \pm 1.18 \mathrm{SD}) \mathrm{fg}$ microcystin cell ${ }^{-1}$ after $8 \mathrm{~d}$ of grazing on M. aeruginosa HUB 524. Hence, the intracellular microcystin contents of Ochromonas sp. were about one order of magnitude lower than the intracellular microcystin contents of the $M$. aeruginosa cells upon which they fed (Table 1).

\section{Functional and numerical response}

Ochromonas sp. showed a very similar functional response irrespective of whether it was feeding on the microcystin-producing wildtype or on the microcystin-de- ficient mutant of Microcystis aeruginosa PCC 7806 (Fig. 4A). The ingestion rate of Ochromonas sp. was well described by the Type 3 functional response model of Eq. (4). We note that the sigmoid shape of the Type 3 functional response is not visible in Fig. 4A, because both axes are plotted on logarithmic scales. The estimated model parameters are summarised in Table 3. The power $x$ had a similar value on both $M$. aeruginosa strains and was significantly $>1$, confirming the Type 3like shape of the functional response of Ochromonas sp. The maximum ingestion rate $I_{\max }$ was slightly but significantly higher on the microcystin-producing wildtype than on the microcystin-deficient mutant (Table 3). The initial increase of the clearance rate with the $M$. 


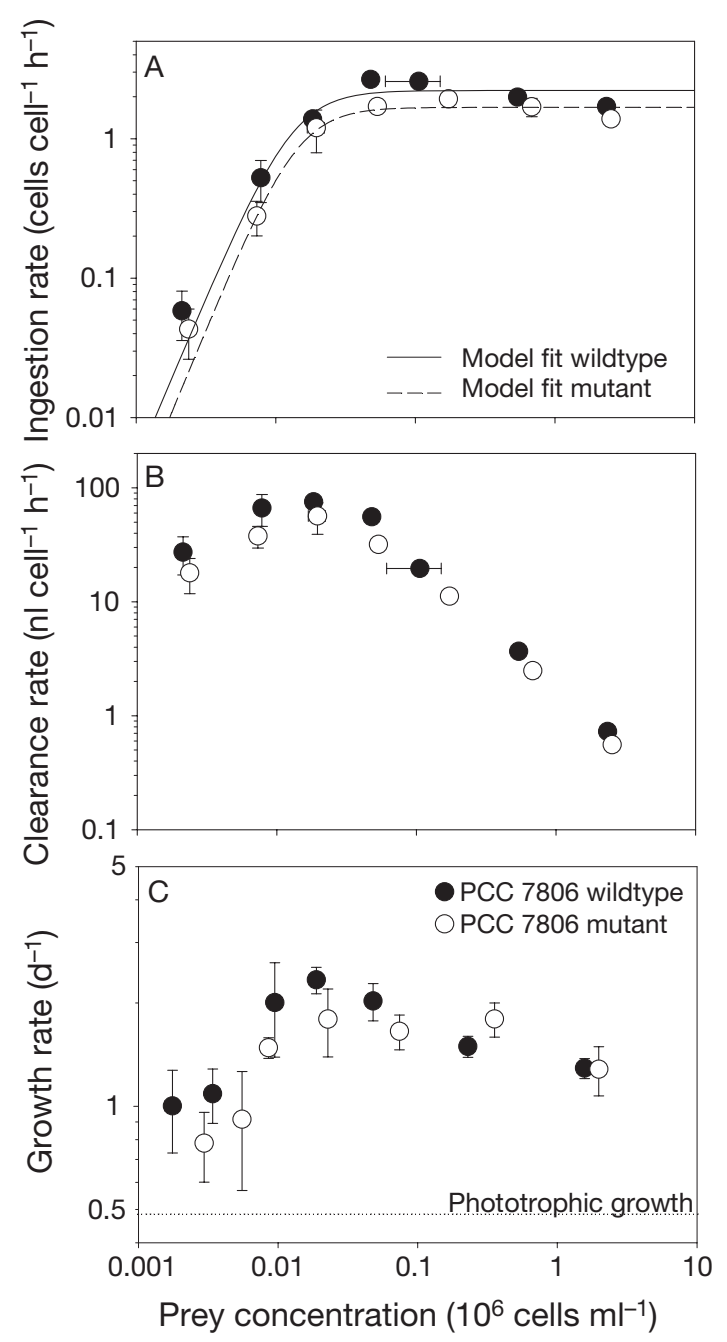

Fig. 4. Ochromonas sp. (A) Ingestion rates, (B) clearance rates and (C) specific growth rates of Ochromonas sp. grazing on the microcystin-producing wildtype and microcystindeficient mutant of Microcystis aeruginosa PCC 7806. Error bars indicate standard deviation. (A) Lines show the fit of the Type 3 functional response model to the ingestion rate data. (C) Dotted horizontal line shows the specific growth rate of Ochromonas sp. when grown photoautotrophically (i.e. without $M$. aeruginosa)

Table 3. Microcystis aeruginosa and Ochromonas sp. Estimated parameter values for the Type 3 functional response model fitted to the ingestion rate data of Ochromonas sp. grazing on the microcystin-producing wildtype and the microcystin-deficient mutant of $M$. aeruginosa PCC 7806. $I_{\max }$ : maximum ingestion rate of Ochromonas sp.; $K$ : half-saturation constant; $x$ : curvature of the sigmoid functional response (Eq. 4). 95\% confidence intervals are given in parentheses

\begin{tabular}{|c|c|c|c|}
\hline $\begin{array}{l}\text { M. aeruginosa } \\
\text { strain }\end{array}$ & ${ }_{\text {(cells cell }}^{-1} \mathrm{~h}^{-1}$ & $\begin{array}{c}K \\
\left(10^{6} \text { cells ml }{ }^{-1}\right)\end{array}$ & $x$ \\
\hline Wildtype & $2.21( \pm 0.25)$ & $0.013( \pm 0.025)$ & $2.37( \pm 0.97)$ \\
\hline Mutant & $1.68( \pm 0.14)$ & $0.014( \pm 0.025)$ & $2.43( \pm 0.85)$ \\
\hline
\end{tabular}

aeruginosa concentration is typical for a Type 3-like functional response (Fig. 4B). The specific growth rate of Ochromonas sp. showed a very similar dependence on $M$. aeruginosa abundance irrespective of whether it was feeding on the microcystin-producing wildtype or the microcystin-deficient mutant (Fig. 4C). The specific growth rate of Ochromonas sp. was much lower when grown photo-autotrophically than when feeding on M. aeruginosa (Fig. 4C).

Abundances of heterotrophic bacteria remained below $1 \%$ of the total biovolume and did not decrease in the grazing treatments relative to the control. The contribution of heterotrophic bacteria to the nutrition of Ochromonas sp. was therefore regarded as negligible in our experiments.

\section{DISCUSSION}

Genetic evidence has shown that microcystins have a long evolutionary history, indicating that cyanobacteria could probably produce microcystins before metazoan grazers such as copepods and cladocerans entered the evolutionary record (Rantala et al. 2004). This makes it unlikely that microcystins have evolved as a defence against metazoans. However, protozoa arrived at the scene much earlier, and microcystins could therefore have evolved as an anti-herbivore defence against protozoan grazers. Our laboratory experiments confirm the strong impact of protozoan grazing by the flagellate Ochromonas sp. on the cyanobacterium Microcystis aeruginosa. The population of Ochromonas sp. grew quickly to large densities and suppressed the abundance of $M$. aeruginosa substantially. This implies that Ochromonas has the potential to impact $M$. aeruginosa populations in natural waters, where Ochromonas is often present at relatively low background densities, but occasionally dominates the phytoplankton (Olrik \& Nauwerck 1993, Van Donk et al. 2009).

Grazing by Ochromonas sp. did not cause an increase in intracellular microcystin content. Therefore, microcystins do not seem to act as an inducible defence against flagellate grazing as has been reported for daphnids (Jang et al. 2003). Since we did not observe any colony formation in the presence of grazers, we cannot totally exclude the possibility that the population densities of Ochromonas sp. in our experiment were too low to cause a response in Microcystis aeruginosa. Colony formation in M. aeruginosa can be induced by Ochromonas grazing (Burkert et al. 2001, Yang et al. 2006). However, these studies used different strains of $M$. aeruginosa, and we could not ascertain that the strains used in our experiments had the ability to form colonies. Our inoculation densities 
were comparable to population densities observed in natural waters (Van Donk et al. 2009), and Ochromonas sp. grew rapidly to higher densities over the course of the experiment. Therefore, if these population densities were too low to induce higher intracellular microcystin contents in our experiments, Ochromonas can not be expected to induce higher intracellular microcystin in natural waters either. Dissolved microcystin concentrations increased significantly due to grazing by Ochromonas sp., probably due to lysis of $M$. aeruginosa cells or excretion of consumed microcystin by Ochromonas sp.

While Ochromonas sp. grew with very high specific growth rates at the start of the experiment, and increased population densities 500- to 1000 -fold, specific growth rates decreased markedly during the course of the experiment (Fig. 2), even though prey densities remained high. This deceleration of the growth rate was not observed in earlier studies using Ochromonas or Poterioochromonas (Cole \& Wynne 1974, Zhang et al. 2008), because these earlier studies used very high inoculation densities of the flagellates, resulting in the disappearance of Microcystis aeruginosa within short periods. What might have caused the reduction of the specific growth rate cannot be answered from our experiments. One possible reason might be the excretion of allelopathic substances by Microcystis (e.g. Sukenik et al. 2002). Another explanation might be the toxic effect of microcystins ingested by Ochromonas sp. This possibility has been further investigated in the functional response experiment.

We used the microcystin-producing wildtype and microcystin-deficient mutant of Microcystis aeruginosa PCC 7806 to investigate the effect of microcystins on the functional and numerical response of Ochromonas sp., since the only difference between these 2 strains is their ability to produce microcystins. The content of other potentially toxic peptides like cyanopeptolins and microviridins (Jungmann 1992, Tonk et al. 2009) should be the same in both of them, while strains of different origin would probably have differed in their contents of many peptides, fatty acids and other cellular constituents.

The ingestion and clearance rates of Ochromonas sp. showed a Type 3 functional response. This shape is explained by an increased foraging effort with increasing prey concentration. In the mixotroph Ochromonas, such a pattern is most likely caused by a trade-off between phototrophic and heterotrophic growth. At low prey densities, mixotrophs will mainly benefit from investments in phototrophic growth, while foraging efforts on prey will contribute less to their total carbon metabolism. Conversely, at high prey densities, the rewards of heterotrophic growth will be high and photosynthesis will therefore become less important. The growth rate achieved under purely autotrophic conditions was much lower than the maximum growth rate of Ochromonas sp. (Fig. 4C), indicating that saturating densities of Microcystis aeruginosa covered at least $80 \%$ of the carbon demand of Ochromonas sp.

Quantitatively, our estimates of the ingestion rates compare well to those of 1.7 cells cell ${ }^{-1} \mathrm{~h}^{-1}$ reported for Ochromonas danica grazing on Microcystis aeruginosa (Cole \& Wynne 1974). The same is true for the clearance rates measured at high prey concentrations. However, the maximum clearance rates of 55 to $75 \mathrm{nl}$ cell $^{-1} \mathrm{~h}^{-1}$ measured in the present study at prey concentrations of about $3 \times 10^{4}$ cells ml ${ }^{-1}$ are much higher than those of 0.6 to $1.9 \mathrm{nl} \mathrm{cell}^{-1} \mathrm{~h}^{-1}$ reported for Poterioochromonas sp. (Zhang et al. 2008) or of 0.7 to $4.1 \mathrm{nl}$ cell $^{-1} \mathrm{~h}^{-1}$ for the heterotrophic flagellate Collodictyon triciliatum grazing on $M$. aeruginosa (Nishibe et al. 2002). The lower clearance rates found in the literature can be explained by the higher prey concentrations used in most other studies and the strong decline of clearance rates with increasing prey concentrations (Fig. 4B).

The intracellular microcystins did not have a negative effect on Ochromonas sp. On the contrary, Ochromonas sp. even showed slightly higher maximum ingestion rates on the microcystin-containing wildtype than on the microcystin-deficient mutant. Whether this is due to a stimulating effect of microcystins (Ou et al. 2005) or to the slightly smaller size of the wildtype strain (Table 1) cannot be answered with certainty. In any case, differences in ingestion rates between the wildtype and its microcystin-deficient mutant were small, and intracellular microcystin, therefore, does not seem to act as a defence against these mixotrophic flagellates.

In addition to mixotrophic flagellates such as Ochromonas, several heterotrophic flagellates have been reported to graze on Microcystis (Nishibe et al. 2002, Park et al. 2003, Kim et al. 2006). Microcystins inhibit the eukaryotic protein phosphatases Types 1 and 2A (MacKintosh et al. 1990) and are therefore toxic to most eukaryotes. However, since the protein phosphatases are located in the cytoplasm, microcystins have to be transported into the cell to cause a toxic effect. Active transport into mammalian hepatocytes has been shown (Dawson 1998), while most other organs remain unaffected, presumably because they do not take up microcystins. A possible explanation for the absence of microcystin toxicity in Ochromonas might therefore be the lack of an uptake system from the lysosomes into the cytoplasm. Furthermore, Ochromonas and Poterioochromonas are both able to degrade microcystins (Zhang et al. 2008, Van Donk et al. 2009), and we did not find any bioaccumulation of microcystins within Ochromonas sp. A rapid degrada- 
tion of the toxins is important in preventing a negative effect on growth. Due to degradation, only a small fraction of the microcystins ingested by Ochromonas sp. was released into the dissolved pool, which is contrary to what would be expected if microcystins were egested directly. Because our cultures were not axenic, there might have been some degradation of dissolved microcystins by bacteria as well.

While our results show that the mixotrophic flagellate Ochromonas sp. is not affected by microcystins, other studies indicate that several species of heterotrophic flagellates are susceptible to microcystins (Christoffersen 1996, Moustaka-Gouni et al. 2006). These contrasting results suggest great variability in the response of different protozoa to microcystin-producing cyanobacteria. In addition to microcystin production, colony formation in Microcystis can also act as a defence against grazers and has been shown to affect Ochromonas clearance rates (Yang et al. 2009). Because Microcystis often forms large colonies, the impact of Ochromonas grazing on single-celled Microcystis strains in our small-scale laboratory experiments cannot be easily extrapolated to natural waters. Further experiments are needed to elucidate the role of Ochromonas grazing on colonial Microcystis populations.

In conclusion, protozoa such as the mixotrophic flagellate Ochromonas can be very effective grazers suppressing the population development of Microcystis aeruginosa. Ochromonas is not negatively affected by the ingested microcystins, nor is microcystin production by $M$. aeruginosa enhanced by Ochromonas grazing. While microcystins are a very powerful defence against many metazoan grazers (DeMott et al. 1991, Lürling 2003), they seem to be less useful against mixotrophic flagellates, and colony formation might be the more effective protection against these very small protozoan grazers.

Acknowledgements. This research was supported by the Water Programme of the Earth and Life Sciences Foundation (ALW), which is subsidized by the Netherlands Organization for Scientific Research (NWO).

\section{LITERATURE CITED}

Boëchat IG, Weithoff G, Krüger A, Gücker B, Adrian R (2007) A biochemical explanation for the success of mixotrophy in the flagellate Ochromonas sp. Limnol Oceanogr 52: 1624-1632

Burkert U, Hyenstrand P, Drakare S, Blomqvist P (2001) Effects of the mixotrophic flagellate Ochromonas sp. on colony formation in Microcystis aeruginosa. Aquat Ecol 35:11-17

> Carmichael WW (2001) Health effects of toxin-producing cyanobacteria: the cyanoHABs. Hum Ecol Risk Assess 7: 1393-1407

Chorus I, Bartram J (1999) Toxic cyanobacteria in water: a guide to their public health consequences, monitoring and management. E \& FN Spon, London

Christoffersen K (1996) Effect of microcystin on growth of single species and on mixed natural populations of heterotrophic nanoflagellates. Nat Toxins 4:215-220

Codd GA (2000) Cyanobacterial toxins, the perception of water quality, and the prioritisation of eutrophication control. Ecol Eng 16:51-60

Cole GT, Wynne MJ (1974) Endocytosis of Microcystis aeruginosa by Ochromonas danica. J Phycol 10:397-410

Dawson RM (1998) The toxicology of microcystins. Toxicon 36:953-962

DeMott WR, Zhang QX, Carmichael WW (1991) Effects of toxic cyanobacteria and purified toxins on the survival and feeding of a copepod and 3 species of Daphnia. Limnol Oceanogr 36:1346-1357

Dittmann E, Neilan BA, Erhard M, von Döhren H, Börner T (1997) Insertional mutagenesis of a peptide synthetase gene that is responsible for hepatotoxin production in the cyanobacterium Microcystis aeruginosa PCC 7806. Mol Microbiol 26:779-787

Fulton RS, Paerl HW (1987a) Effects of colonial morphology on zooplankton utilization of algal resources during bluegreen algal (Microcystis aeruginosa) blooms. Limnol Oceanogr 32:634-644

Fulton RS, Paerl HW (1987b) Toxic and inhibitory effects of the blue-green alga Microcystis aeruginosa on herbivorous zooplankton. J Plankton Res 9:837-855

> Gustafsson S, Rengefors K, Hansson LA (2005) Increased consumer fitness following transfer of toxin tolerance to offspring via maternal effects. Ecology 86:2561-2567

- Heinbokel JF (1978) Studies on the functional role of tintinnids in the Southern California Bight. 1. Grazing and growth rates in laboratory cultures. Mar Biol 47:177-189

Holling CS (1959) The components of predation as revealed by a study of small mammal predation of the European pine sawfly. Can Entomol 91:293-320

Huisman J, Matthijs HCP, Visser PM (2005) Harmful cyanobacteria. Springer, Berlin

Jang MH, Ha K, Joo GJ, Takamura N (2003) Toxin production of cyanobacteria is increased by exposure to zooplankton. Freshw Biol 48:1540-1550

Jöhnk KD, Huisman J, Sharples J, Sommeijer B, Visser PM, Stroom JM (2008) Summer heatwaves promote blooms of harmful cyanobacteria. Glob Change Biol 14:495-512

Jungmann D (1992) Toxic compounds isolated from Microcystis PCC7806 that are more active against Daphnia than two microcystins. Limnol Oceanogr 37:1777-1783

Kilham SS, Kreeger DA, Lynn SG, Goulden CE, Herrera L (1998) COMBO: a defined freshwater culture medium for algae and zooplankton. Hydrobiologia 377:147-159

> Kim BR, Nakan S, Kim BH, Han MS (2006) Grazing and growth of the heterotrophic flagellate Diphylleia rotans on the cyanobacterium Microcystis aeruginosa. Aquat Microb Ecol 45:163-170

Kiørboe T (2008) A mechanistic approach to plankton ecology. Princeton University Press, Princeton, NJ

Long BM, Jones GJ, Orr PT (2001) Cellular microcystin content in N-limited Microcystis aeruginosa can be predicted from growth rate. Appl Environ Microbiol 67:278-283

Lürling M (2003) Daphnia growth on microcystin-producing and microcystin-free Microcystis aeruginosa in different mixtures with the green alga Scenedesmus obliquus. Limnol Oceanogr 48:2214-2220

Lürling M, Van Donk E (1997) Morphological changes in Scenedesmus induced by infochemicals released in situ from zooplankton grazers. Limnol Oceanogr 42:783-788 
MacKintosh C, Beattie KA, Klumpp S, Cohen P, Codd GA (1990) Cyanobacterial microcystin-LR is a potent and specific inhibitor of protein phosphatases 1 and 2A from both mammals and higher plants. FEBS Lett 264:187-192

Moustaka-Gouni M, Vardaka E, Michaloudi E, Kormas KA and others (2006) Plankton food web structure in a eutrophic lake with a history of toxic cyanobacterial blooms. Limnol Oceanogr 51:715-727

> Nishibe Y, Kawabata Z, Nakano S (2002) Grazing on Microcystis aeruginosa by the heterotrophic flagellate Collodictyon triciliatum in a hypertrophic pond. Aquat Microb Ecol 29:173-179

Olrik K, Nauwerck A (1993) Stress and disturbance in the phytoplankton community of a shallow, hypertrophic lake. Hydrobiologia 249:15-24

$>$ Ou D, Song L, Gan N, Chen W (2005) Effects of microcystins on and toxin degradation by Poterioochromonas sp. Environ Toxicol 20:373-380

Paerl HW, Huisman J (2008) Blooms like it hot. Science 320:57-58

Park S, Brett MT, Müller-Navarra DC, Shin SC, Liston AM, Goldman CR (2003) Heterotrophic nanoflagellates and increased essential fatty acids during Microcystis decay. Aquat Microb Ecol 33:201-205

Rantala A, Fewer DP, Hisbergues M, Rouhiainen L, Vaitomaa J, Börner T, Sivonen K (2004) Phylogenetic evidence for the early evolution of microcystin synthesis. Proc Natl Acad Sci USA 101:568-573

Real LA (1977) Kinetics of functional response. Am Nat 111: 289-300

Sukenik A, Eshkol R, Livne A, Hadas O and others (2002) Inhibition of growth and photosynthesis of the dinoflagel-

Editorial responsibility: Urania Christaki, Wimereux, France late Peridinium gatunense by Microcystis sp. (cyanobacteria): a novel allelopathic mechanism. Limnol Oceanogr 47:1656-1663

- Tonk L, Welker M, Huisman J, Visser PM (2009) Production of cyanopeptolins, anabaenopeptins, and microcystins by the harmful cyanobacteria Anabaena 90 and Microcystis PCC 7806. Harmful Algae 8:219-224

Van Donk E, Cerbin S, Wilken S, Helmsing NR, Ptacnik R, Verschoor AM (2009) The effect of a mixotrophic chrysophyte on toxic and colony-forming cyanobacteria. Freshw Biol 54:1843-1855

von Elert E, Martin-Creuzburg D, Le Coz JR (2003) Absence of sterols constrains carbon transfer between cyanobacteria and a freshwater herbivore (Daphnia galeata). Proc Biol Sci 270:1209-1214

Wacker A, von Elert E (2001) Polyunsaturated fatty acids: evidence for non-substitutable biochemical resources in Daphnia galeata. Ecology 82:2507-2520

Wiedner C, Visser PM, Fastner J, Metcalf JS, Codd GA, Mur LR (2003) Effects of light on the microcystin content of Microcystis strain PCC 7806. Appl Environ Microbiol 69:1475-1481

> Yang Z, Kong FX, Shi XL, Cao HS (2006) Morphological response of Microcystis aeruginosa to grazing by different sorts of zooplankton. Hydrobiologia 563:225-230

Yang Z, Kong FX, Yang Z, Zhang M, Yu Y, Qian SQ (2009) Benefits and costs of the grazer induced colony formation in Microcystis aeruginosa. Int J Limnol 45:203-208

Zhang X, Hu HY, Hong Y, Yang J (2008) Isolation of a Poterioochromonas capable of feeding on Microcystis aeruginosa and degrading microcystin-LR. FEMS Microbiol Lett 288:241-246

Submitted: August 18, 2009; Accepted: December 15, 2009 Proofs received from author(s): April 6, 2010 